\title{
On Zero Modes and the Vacuum Problem - A Study of Scalar Adjoint Matter in Two-Dimensional Yang-Mills Theory via Light-Cone Quantisation.
}

\author{
Alex C. Kalloniatis \\ Max-Planck-Institut für Kernphysik \\ D-69029 Heidelberg
}

15 August, 1995.

\begin{abstract}
SU(2) Yang-Mills Theory coupled to massive adjoint scalar matter is studied in $(1+1)$ dimensions using Discretised Light-Cone Quantisation. This theory can be obtained from pure Yang-Mills in 2+1 dimensions via dimensional reduction. On the light-cone, the vacuum structure of this theory is encoded in the dynamical zero mode of a gluon and a constrained mode of the scalar field. The latter satisfies a linear constraint, suggesting no nontrivial vacua in the present paradigm for symmetry breaking on the light-cone. I develop a diagrammatic method to solve the constraint equation. In the adiabatic approximation I compute the quantum mechanical potential governing the dynamical gauge mode. Due to a condensation of the lowest momentum modes of the dynamical gluons, a centrifugal barrier is generated in the adiabatic potential. In the present theory however, the barrier height appears too small to make any impact in this model. Although the theory is superrenormalisable on naive powercounting grounds, the removal of ultraviolet divergences is nontrivial when the constrained mode is taken into account. The open aspects of this problem are discussed in detail.
\end{abstract}

Preprint: MPI-H-V29-1995 


\section{Introduction}

For some years now intensive work has gone into developing light-cone field theoretic methods [1, 2, 3, 4] for solving the problem of the hadron spectrum from Quantum Chromodynamics. The fundamental advantage of Dirac's 'front form' Hamiltonian framework [5] is the simple vacuum structure and the (related) positivity of the momentum operator. With these one can foresee a picture of hadrons emerging from a diagonalisation of the light-cone Hamiltonian consistent within the intuitive picture of the constituent quark model (see also [6]).

However, two problems have been the stumbling block to this program: renormalisation and the vacuum problem. My main concern in the present work is the latter problem. Ironically, for all the advantages of a simple vacuum for understanding hadron structure, the sophisticated field theoretic picture of QCD as a gauge field theory demands some non-trivial vacuum structure associated with the non-Abelian group topology. In other words, the constituent quark picture works well, but it doesn't work everywhere in stronginteractions. A reasonable 'solution' to QCD must encompass both features.

A scenario in which vacuum structure and the advantages of the front-form could sensibly co-exist was recently put forward by Robertson [7]. In the infra-red regularisation achieved by Discretised Light-Cone Quantisation (DLCQ) of a given field theory, some zero mode field operators are not dynamical field quanta, thus preserving vacuum simplicity, but rather satisfy constraint equations [8]. In some simpler models in which symmetries are known to be spontaneously broken these constraints happen to possess multiple solutions. The light-cone symmetry breaking paradigm thus associates each of these solutions with a particular vacuum choice in the instant-form treatment of the same theory. Thus for example, the scalar $\phi^{4}$ model in $1+1$ dimensions, also studied by [9], has a zero mode satisfying a cubic equation. One of the solutions corresponds to the trivial (unbroken phase) vacuum. The other two reflect the broken phase of the theory. Thus, for example, one could do perturbation theory about the classical solution in either phase [7]. Alternately, van de Sande et al. [10, 11, 12] thoroughly show how the Tamm-Dancoff method can be brought to bear on this problem with a determination of condensates and the critical coupling. Another approach using the $1 / N$ expansion for analogous models has been developed by [13] with similar physics emerging.

Turning to QCD, the paradigm appears difficult to apply. The phenomena one seeks are: 1) $\theta$-vacua or their analogue, 2) spontaneous (anomalous) breaking of chiral (scale) symmetry leading to the appearance of condensates in the nonperturbative vacuum $|\Omega\rangle$ such as $\frac{\alpha_{s}}{\pi}\left\langle\Omega\left|G_{\mu \nu}^{a} G^{a \mu \nu}\right| \Omega\right\rangle \sim 10^{-2}$. The value ascribed here leads to a parametrisation of hadronic resonances via QCD Sum Rules [14]. All these effects are related to non-trivial 
gluonic configurations in the ground state. If the above paradigm is to be relevant, then constrained zero modes of the gluons should play a role. Such objects indeed occur in nonAbelian gauge theory in $(3+1)$ dimensions in DLCQ as shown in the remarkable paper of Franke et al. [15]. Working with SU(2) pure glue theory, they introduced a modified light-cone gauge consistent with the space compactification,

$$
\partial_{-} A^{+}=0
$$

with an additional colour rotation rendering the zero mode of $A^{+}$diagonal in colour space. It turns out that a constrained zero mode appears only in the corresponding diagonal component of the transverse gluons. There is only one constraint equation for the zero mode of the field $A_{\perp}^{3}$. This equation turns out to be linear and thus there is a unique solution [15]. How 'multiple vacua' now emerge in light of this is not clear.

The additional feature in gauge theory is the presence of an additional, dynamical, zero mode. In the case of Franke et al., it manifests itself as the surviving piece of the $A^{+}$ gauge potential. Dynamical zero modes in general mean the naive argument for vacuum triviality in the front form does not apply. The key questions are: just how nontrivial is the vacuum, can one bring it under control, and do the zero modes produce the desired vacuum structure?

The context in which I will try to answer these questions is that of SU(2) YangMills theory coupled to scalar adjoint matter in $(1+1)$ dimensions. As shown elsewhere, this theory can be regarded as the dimensional reduction of pure gauge theory in $(2+1)$ dimensions [16]. The zero mode structure of this theory was described in an earlier work [17] wherein it was shown that, in the same gauge as Franke et al., there is one dynamical and one constrained zero mode. The spectrum of the theory has been further studied in [18]. The approach taken in [17] to the dynamical mode was similar to that of, for example, Manton [19] in the Schwinger model or of Lenz et al. [20] for the case of QCD $(1+1)$ with adjoint fermions. The latter is based on a rather new method of gauge-fixing in the Weyl gauge [21]. What was lacking in [17] was a solution to the constraint equation, which the present work sets out to complete. In this respect, this work is very much a part in the series of works [22, 23].

The approach for the dynamical, or 'gauge', mode in the above works is in the spirit of the adiabatic approximation. The gauge mode part of the Hamiltonian is not in fact separable from the Fock part. Nonetheless, the gauge mode is 'frozen' and the vacuum of the particle sector determined. The dynamics of the gauge mode is then solved for in the vacuum of the particle sector. In this theory, there is no $Z$-vacuum degeneracy. Rather, one expects a two-fold $Z_{2}$ degeneracy as evident from the global symmetries of the 'adiabatic potential' which controls the dynamics of the gauge zero mode. It is not 
clear from the literature whether one should expect any condensate phenomena in this specific model.

In the previous work [17], this potential was computed in the absence of the constrained zero mode. It was found to contain logarithmic divergences for which the counterterms, at the time, were not evident. However, the result for the cutoff independent part was of a flat approximately square well structure. This is rather unlike the result with adjoint fermions 20] where a centrifugal barrier appears in the adiabatic potential. It was already noted for scalar matter [17], that the absence of fermions and transverse momenta meant insufficient degrees of freedom for some composite operator to acquire a condensate. However, it is at least desirable to see whether there is sufficient structure in the gluonic ground state to provide the 'seeding' for such non-triviality when further modes are included.

In the present work I therefore address the two main problems which, in [17, remained untreated: solving the constraint equation and the renormalisation problems in the potential. For the former, I develop a diagrammatic representation which will guide a solution of the constraint. The most significant aspect of the solution is an iteration to all orders of two-point insertions in the constraint solution. For the second problem, I am able to report partial success but difficulties remain. In particular, in the absence of the constrained zero mode the divergences in the potential can be naturally handled with mass renormalisation. Finally, I study the inclusion of the constraint in the potential. There remain logarithmic divergences which in the present work are subtracted by hand. In the renormalised potential one observes that a condensation of the lowest momentum mode of the scalar field, achieved by the sum to all orders of the insertion in the constraint solution, leads indeed to the appearance of a distinct, albeit small, potential barrier. The barrier is highest in the case of the renormalised mass of the 'gluons' being zero. If an exact $Z_{2}$ degeneracy is to arise in the continuum limit, this barrier would have to become impenetrable. This does not appear to happen here. In the final section, I discuss in some detail the open problem of the remaining logarithmic divergences. There is a brief summary at the end. Notation and other details are relegated to the Appendices.

\section{Review of Scalar Adjoint Matter and SU(2) Yang-Mills}

The model-theory I work with is $(1+1)$ dimensional non-Abelian gauge theory covariantly coupled to massive scalar adjoint matter

$$
\mathcal{L}=\operatorname{Tr}\left(-\frac{1}{2} \mathbf{F}^{\alpha \beta} \mathbf{F}_{\alpha \beta}+\mathbf{D}^{\alpha} \Phi \mathbf{D}_{\alpha} \Phi-\mu_{0}^{2} \Phi^{2}\right)
$$

The field strength tensor and covariant derivative $\mathbf{D}_{\alpha}$ are respectively defined by

$$
\mathbf{D}^{\alpha}=\partial^{\alpha}+i g\left[\mathbf{A}^{\alpha}, \cdot\right] \quad \text { and } \quad \mathbf{F}^{\alpha \beta}=\partial^{\alpha} \mathbf{A}^{\beta}-\partial^{\beta} \mathbf{A}^{\alpha}+i g\left[\mathbf{A}^{\alpha}, \mathbf{A}^{\beta}\right] \text {. }
$$


As in [17, I represent field matrices in a colour helicity basis:

$$
\Phi=\tau^{3} \varphi_{3}+\tau^{+} \varphi_{+}+\tau^{-} \varphi_{-} .
$$

Treatments of this theory in DLCQ have been given in Refs. [16, 17]. The equations of motion are

$$
\mathbf{D}_{\beta} \mathbf{F}^{\beta \alpha}=g \mathbf{J}^{\alpha}, \text { with } \quad \mathbf{J}^{\alpha}=-i\left[\Phi, \mathbf{D}^{\alpha} \Phi\right], \quad \text { and } \quad\left(\mathbf{D}^{\alpha} \mathbf{D}_{\alpha}+\mu_{0}^{2}\right) \Phi=0
$$

Note that the 'matter current' $\mathbf{J}^{\alpha}$ is not conserved, $\partial_{\alpha} \mathbf{J}^{\alpha} \neq 0$, whereas the total 'gluon current' $\mathbf{J}_{\mathrm{G}}^{\alpha}=\mathbf{J}^{\alpha}-i\left[\mathbf{F}^{\alpha \beta}, \mathbf{A}_{\beta}\right]$ is conserved. I use the light-cone Coulomb gauge $\partial_{-} \mathbf{A}^{+}=0$, which preserves the zero mode of $\mathbf{A}^{+}$. Then a single rotation in colour space suffices to diagonalise the $\mathrm{SU}(2)$ colour matrix $\mathbf{A}^{+}=A_{3}^{+} \tau_{3}$. Finally, the diagonal zero mode of $\mathbf{A}^{-}\left(x_{0}^{+}\right)$can be gauged away [24] at some fixed light-cone time $x_{0}^{+}$. For writing the Hamiltonian, it is convenient to choose this time as $x_{0}^{+}=0$, the null-plane initial value surface on which we specify the independent fields. The quantum mode $A_{3}^{+}$has a conjugate momentum $p \equiv \delta L / \delta\left(A_{3}^{+}\right)=2 L \partial_{+} A_{3}^{+}$and satisfies the commutation relation

$$
\left[A_{3}^{+}, p\right]=\left[A_{3}^{+}, 2 L \partial_{+} A_{3}^{+}\right]=i
$$

As in [17], I shall work in Schrödinger representation for this quantum mechanical degree of freedom. In the following it will be useful to invoke the dimensionless combination

$$
z \equiv \frac{g A_{3}^{+} L}{\pi}
$$

There are additional global symmetries which can be seen in terms of this mode. First, Gribov copies [31, 32] correspond to shifts $z \rightarrow z+2 n, n \in Z$, see Appendix B. Shifts $z \rightarrow z+(2 n+1), n \in Z$ are 'copies' generated by the group of centre conjugations of $\mathrm{SU}(2)$, namely $Z_{2}$ symmetry 25 . The finite interval $0<z<1$ is called the fundamental modular domain, see for example [26]. Two further symmetries are important: Weyl reflection, $z \rightarrow-z$ and the composition of a reflection and centre transformation leading to a symmetry of the theory under $z \rightarrow(1-z)$. After selection of the fundamental domain, this last is the only remnant symmetry leading to symmetry under reflection about $z=\frac{1}{2}$. To further prepare the reader, it will later become convenient to switch to a variable $\zeta=\left(z-\frac{1}{2}\right)$ in the fundamental modular domain.

The diagonal component of the hermitian colour matrix $\Phi$ is $\varphi_{3}$. The quantisation, with the exception of the zero mode $\stackrel{\circ}{\varphi}_{3}=a_{0} / \sqrt{4 \pi}$, is straightforward. At $x^{+}=0$, I expand in momentum modes

$$
\varphi_{3}\left(x^{-}\right)=\frac{a_{0}}{\sqrt{4 \pi}}+\frac{1}{\sqrt{4 \pi}} \sum_{l=1}^{\infty}\left(a_{l} w_{l} \mathrm{e}^{-i l \frac{\pi}{L} x^{-}}+a_{l}^{\dagger} w_{l} \mathrm{e}^{+i l \frac{\pi}{L} x^{-}}\right)
$$


where $w_{l}=1 / \sqrt{l}$. Note that I will reserve $l, l^{\prime}, l_{1}, \ldots$ for the nonzero integer valued momenta of the real scalar field. The momentum field conjugate to $\varphi_{3}$ is $\pi^{3}=\partial_{-} \varphi_{3}$. The quantum commutation relation at equal $x^{+}$for the normal modes is given in Appendix $\mathrm{C}$. However, it leads to the Fock commutator $\left[a_{l}, a_{l^{\prime}}^{\dagger}\right]=\delta_{l, l^{\prime}}\left(l, l^{\prime}>0\right)$. Sometimes it will be convenient to write the Kronecker $\delta_{l, l^{\prime}}$ as $\delta_{l}^{l^{\prime}}$. As the zero mode of $\pi_{3}$ vanishes, the zero mode of $\varphi$ is not a degree of freedom, but will turn out to satisfy a constraint which is the main point of this paper.

The off-diagonal components of $\Phi$ are complex fields with $\varphi_{+}\left(x^{-}\right)=\varphi_{-}^{\dagger}\left(x^{-}\right)$. The momentum conjugate to $\varphi_{-}$is $\pi^{-}=\left(\partial_{-}+i g v\right) \varphi_{+}$. The other conjugate pair is obtained simply by hermitian conjugation. A way of quantizing this field which is not complicated by the large gauge transformations was discussed in [17]. The expansion is over half-integer momenta but in a manner consistent with periodic boundary conditions, namely

$$
\varphi_{-}(x)=\frac{\mathrm{e}^{+i m_{0} \frac{\pi}{L} x^{-}}}{\sqrt{4 \pi}} \sum_{m=\frac{1}{2}}^{\infty}\left(b_{m} u_{m} \mathrm{e}^{-i m \frac{\pi}{L} x^{-}}+d_{m}^{\dagger} v_{m} \mathrm{e}^{+i m \frac{\pi}{L} x^{-}}\right) .
$$

where $u_{m}(z)=1 / \sqrt{m+\zeta}$ and $v_{m}(z)=1 / \sqrt{m-\zeta}$. The objects $m_{0}$ and $\zeta$ are functions of $z$, defined by $m_{0}(z)=($ integer part of $z)-\frac{1}{2}, \zeta(z)=z-m_{0}(z)$. They satisfy the relations

$$
m_{0}(z+1)=m_{0}(z)+1, \quad m_{0}(-z)=-m_{0}(z), \quad \zeta(z+1)=\zeta(z), \quad \zeta(-z)=-\zeta(z) .
$$

The domain interval is now $-\frac{1}{2}<\zeta(z)<\frac{1}{2}$ for all values of $z$. For the fundamental domain $m_{0}=-\frac{1}{2}$, but the specific choice no longer matters. The Fock modes then obey boson commutation relations

$$
\left[b_{n}, b_{m}^{\dagger}\right]=\left[d_{n}, d_{m}^{\dagger}\right]=\delta_{n}^{m}, \quad \text { and } \quad\left[b_{n}, d_{m}\right]=\left[b_{n}, d_{m}^{\dagger}\right]=0
$$

Finally one notes that a large gauge transformation $z \rightarrow z+1$ produces only $m_{0} \rightarrow m_{0}+1$ and thus only a change of the overall phase in Eq.(2.9). Most importantly it does not change the particle-hole assignment and thus the Fock vacuum defined with respect to $b_{m}$ and $d_{m}$ is invariant under these transformations.

The only modes not discussed thus far are the $A^{-}$fields. As is known for the Coulomb gauge, they are redundant variables obtained from implementing Gauss' law strongly. Explicitly, Gauss' law reads

$$
-\partial_{-}^{2} A_{3}=g J_{3}^{+}, \quad-\left(\partial_{-}+i g A_{3}^{+}\right)^{2} A_{+}=g J_{+}^{+},
$$

and the hermitian conjugate of the latter with $\left(J_{+}^{+}\right)^{\dagger} \equiv J_{-}^{+}$. The currents are, explicitly,

$$
J_{3}^{+}=\frac{1}{i}\left(\varphi_{+} \pi_{-}-\varphi_{-} \pi_{+}\right)_{s} \text { and } J_{+}^{+}=\frac{1}{i}\left(\varphi_{3} \pi_{+}-\varphi_{+} \pi_{3}\right)_{s} .
$$


The index $s$ indicates noncommuting operators in this product which must be symmetrised in order to preserve hermiticity. The Eqs. 2.12) are trivially soluble for the fields $A^{-}$ subject to the following exception. The first of Eq.(2.12) can be solved only if the zero mode $\left\langle J_{3}^{+}\right\rangle_{\circ} \equiv Q_{0}$ on the r.h.s vanishes. This cannot be satisfied as an operator, but must be used to select out physical states, i.e. $Q_{0} \mid$ phys $\rangle \equiv 0$. In second-quantised form this gives

$$
\left.\left.Q_{0} \mid \text { phys }\right\rangle=\sum_{m=\frac{1}{2}}^{\infty}\left(b_{m}^{\dagger} b_{m}-d_{m}^{\dagger} d_{m}\right) \mid \text { phys }\right\rangle=0 .
$$

States satisfying this have the same total number of "b" and "d" particles. The resemblance to the electric-charge neutrality condition is because the residual global gauge symmetry group factored out of the Hilbert space is Abelian. Evidently, the b-modes carry "charge" 1 , the d-modes charge -1 and the a-modes charge 0 .

\section{A Diagrammar for the Zero Mode Problem}

The origin of the constraint equation for $a_{0}$ has already been discussed in [17]: the zero mode of the diagonal part of Eq.(2.5), $8 \pi \operatorname{Tr}\left\langle\tau^{3}\left(\mathbf{D}^{\alpha} \mathbf{D}_{\alpha}+\mu_{0}^{2}\right) \Phi\right\rangle_{\circ} / g^{2}=0$. After some algebra this gives

$$
i\left\langle\varphi_{+} \frac{1}{\left(\partial_{-}-i g v\right)} J_{-}^{+}-\varphi_{-} \frac{1}{\left(\partial_{-}+i g v\right)} J_{+}^{+}\right\rangle_{0, s}-\frac{\mu_{0}^{2}}{g^{2} \sqrt{4 \pi}} a_{0}=0 .
$$

In the absence of a mass term, the constraint in its 'full glory' was shown in 27]. To proceed here I first introduce the dimensionless ratio of boson mass and coupling

$$
\rho_{0} \equiv \frac{4 \pi \mu_{0}^{2}}{g^{2}}
$$

Next I introduce the following notation:

$$
\begin{aligned}
\Delta_{m}(\zeta) & =\frac{1}{2(m+\zeta)} \\
\Gamma_{0}^{l m n}(\zeta) & =\left[\left(\frac{w_{l}}{v_{n}}+\frac{v_{n}}{w_{l}}\right) u_{m}^{3}+\left(\frac{w_{l}}{u_{m}}+\frac{u_{m}}{w_{l}}\right) v_{n}^{3}\right] \\
\Gamma_{1}^{l m n}(\zeta) & =-\left[\left(\frac{w_{l}}{u_{m}}-\frac{u_{m}}{w_{l}}\right) u_{n}^{3}+\left(\frac{w_{l}}{u_{n}}+\frac{u_{n}}{w_{l}}\right) u_{m}^{3}\right]
\end{aligned}
$$

The constraint equation turns out to be

$$
\begin{gathered}
\sum_{n=\frac{1}{2}}^{\infty}\left[\Delta_{n}(\zeta)\left(b_{n}^{\dagger} b_{n} a_{0}\right)_{s}+\Delta_{n}(-\zeta)\left(d_{n}^{\dagger} d_{n} a_{0}\right)_{s}\right]+\rho_{0} a_{0}= \\
\sum_{l=1}^{\infty} \sum_{m, n=\frac{1}{2}}^{\infty}\left[\delta_{m+n}^{l} \Gamma_{0}^{l m n}(\zeta)\left(a_{l}^{\dagger} b_{m} d_{n}+a_{l} b_{m}^{\dagger} d_{n}^{\dagger}\right)+\right. \\
\left.\delta_{l+m}^{n} \Gamma_{1}^{l m n}(\zeta)\left(a_{l}^{\dagger} b_{m}^{\dagger} b_{n}+a_{l} b_{m} b_{n}^{\dagger}\right)+\delta_{l+m}^{n} \Gamma_{1}^{l m n}(-\zeta)\left(a_{l}^{\dagger} d_{m}^{\dagger} d_{n}+a_{l} d_{m} d_{n}^{\dagger}\right)\right] .
\end{gathered}
$$


One observes that, the dynamically independent composite operators on the left-hand side of the equation are two-body operators - propagators - while on the right-hand side they are three-body operators, namely interaction vertices. This suggests diagrammatic rules for the various quantities in this expression which are represented in Fig.(1). Additional rules are as follows:

1. Matrix elements are composed by attaching legs to the right and left of the blob representing the operator.

2. Closed loops represent a sum over all positive integer or half-integer momenta.

3. Detached diagrams represent multiplication of the corresponding expressions.

4. Hermitian conjugation is achieved by reflection of the diagram while keeping the sense of arrows the same.

5. Charge conjugation is achieved by reversing the direction of the arrows.

Evidently, these are quite similar to Feynman rules but describe the building of more than just S-matrix elements. Here I will use them to relate matrix elements of the constrained zero mode as determined by the constraint equation.

To illustrate the use of the rules, the object

$$
\Delta_{m}(\zeta)\left\langle 0\left|b_{m} d_{n} a_{0} a_{l}^{\dagger}\right| 0\right\rangle
$$

describes a matrix element of $a_{0}$ multiplied by a propagator factor. Diagrammatically, this is represented by Fig.(2).

Next, one can represent matrix elements of the constraint equation in a particular sector. For example, taking $\langle 0| a_{l}$ on the left and $b_{m}^{\dagger} d_{n}^{\dagger}|0\rangle$ on the right and using commutators where possible, one obtains an equation which can be represented diagrammatically as shown in Fig.(3). The mass term is evidently the fifth term. Similar diagrammatic equations can be generated by sandwiching the constraint between higher particle states, and the intuitive principal for their construction is straightforward:

1. On the right hand side, join up the incoming and outgoing lines with the three available vertices in all possible permutations, with the remaining lines running through as 'spectators'.

2. On the left hand side, assign propagator legs once only to all 'incoming/outgoing' b- or d-boson legs, attach all possible permutations of tadpoles and loops.

The following features can be observed in Fig.(3) and the original equation Eq.(3.18). 


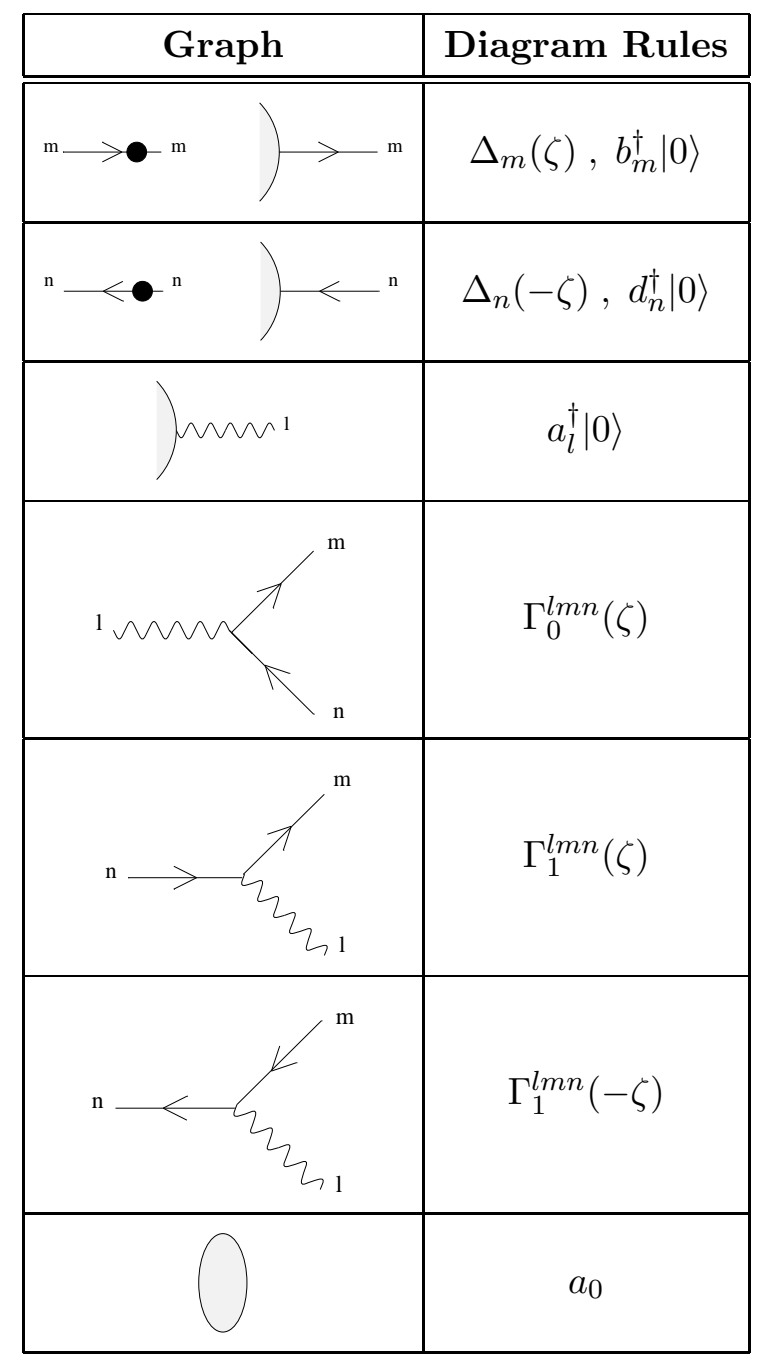

Figure 1: Diagrammatic Rules for the objects appearing in the constraint equation Eq.(3.18). Hermitian conjugation involves reflection through the vertical plane without changing senses of arrows. Further rules are explained in the text.

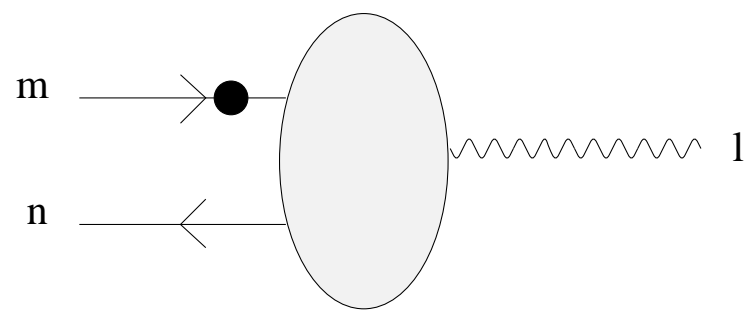

Figure 2: Example of use of the diagrammatic rules for the product of a matrix element and a propagator: $\Delta_{m}(\zeta)\left\langle 0\left|b_{m} d_{n} a_{0} a_{l}^{\dagger}\right| 0\right\rangle$. 

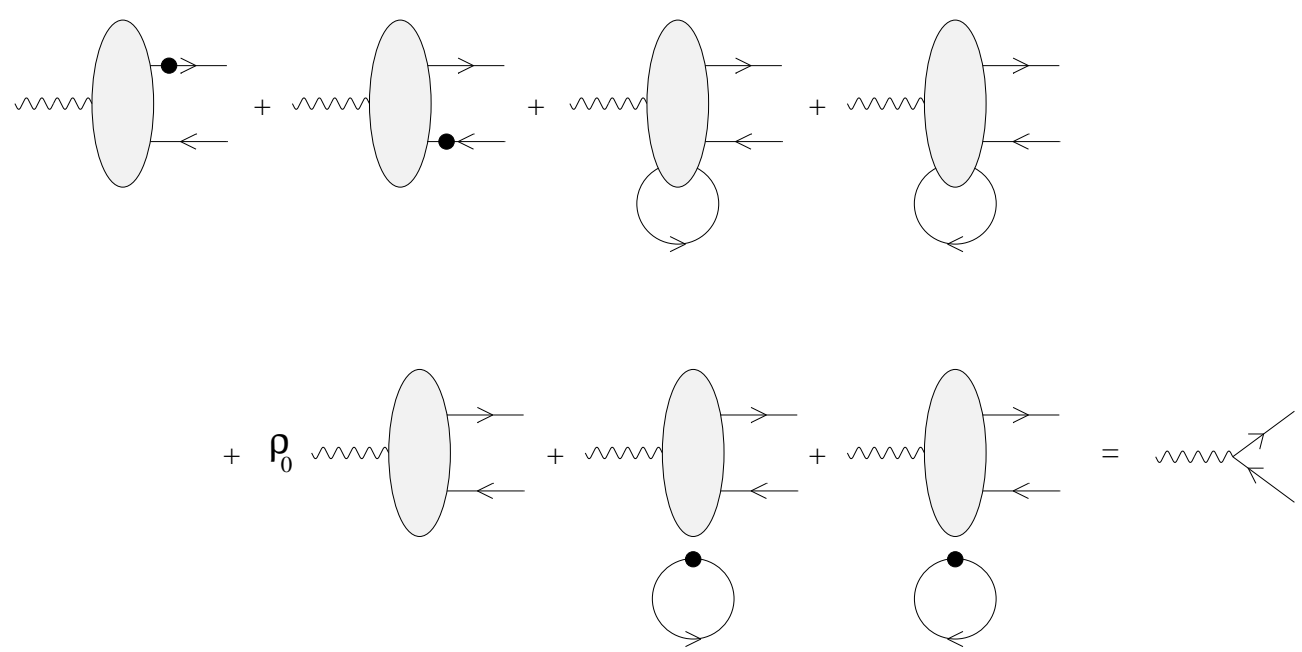

Figure 3: The constraint equation Eq.(3.18) evaluated between $\langle 0| a_{l}$ and $b_{m}^{\dagger} d_{n}^{\dagger}|0\rangle$ depicted diagrammatically.

Firstly, the equation involves matrix elements of the constrained mode in the 5-body sector (two particles 'in', three 'out'). To get at these one takes matrix elements of the constraint equation in this higher sector. This I will show below. Naturally, this in turn involves matrix elements from the 7-body sector (three 'in', four 'out'). This sequence carries on ad infinitum. Thus all the particle sectors appear related to each other via the constrained mode which is playing the role of an effective interaction. The question is how complicated is this coupling of sectors?

One can rule out any coupling of the vacuum sector into the hierarchy. The VEV $\left\langle 0\left|a_{0}\right| 0\right\rangle=0$ because under charge conjugation円 $a_{0} \rightarrow-a_{0}$ while the vacuum is even.

In addition, the following commutation relations for $a_{0}$ must be satisfied:

$$
\begin{aligned}
{\left[a_{0}, Q_{0}\right] } & =0 \\
{\left[a_{0}, P^{+}\right] } & =0 .
\end{aligned}
$$

These can be used to further reduce the number of non-zero matrix elements.

Momentum conservation through the vertices will mean that for many sectors the right-hand side of the hierarchy of coupled equations will vanish. It is difficult to prove generally, but the system of linear coupled equations can be shown to be nonsingular for simple examples. Thus matrix elements of $a_{0}$ which cannot be described in terms of the basic vertices can be argued to vanish. An example is the matrix element in Fig.(甸).

\footnotetext{
${ }^{1}$ For a colour matrix $\boldsymbol{\Phi}$, charge conjugation is represented by $\Phi_{i j} \rightarrow-\Phi j i$, where $(i, j)$ are the indices of the matrix in colour space 33].
} 


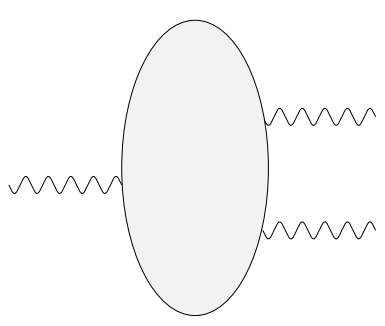

Figure 4: An example of a matrix element vanishing because the operator cannot be replaced by one of the basic vertices in the constraint equation.

Renormalisation of the Constraint. The graphs in Fig.(3) with detached bubbles are logarithmically divergent. I introduce a cutoff regulator $\Lambda$ in the momentum sums so that these terms become

$$
\sum_{n=1 / 2}^{\Lambda}\left(\Delta_{n}(\zeta)+\Delta_{n}(-\zeta)\right) a_{0} \sim(\ln \Lambda+\text { finite }) a_{0}
$$

This divergence can be absorbed into the mass term by a subtraction of the sum for $\zeta=0$, namely with physical mass $\rho$ given by

$$
\begin{aligned}
\rho & =\rho_{0}+4 \sum_{m=\frac{1}{2}}^{\Lambda} \Delta_{m}(0) \\
& =\rho_{0}+4\left[\frac{1}{2}\left(\gamma_{E}+\ln \Lambda\right)+\ln 2+\mathcal{O}(1 / \Lambda)\right] .
\end{aligned}
$$

One can verify that this is the same renormalisation used to remove all cutoff dependence in the two-particle bound state equation in, for example, [18]. A similar relationship between the two renormalisations has already been observed in the two-dimensional $\phi^{4}$ theory by [10]. As a consequence, the left hand side of the original constraint now takes the following form

$$
\sum_{m=\frac{1}{2}}^{\Lambda}\left[\Delta_{m}(\zeta)\left(b_{m}^{\dagger} b_{m} a_{0}+b_{m}^{\dagger} a_{0} b_{m}+a_{0} b_{m}^{\dagger} b_{m}+\left(b_{m} a_{0} b_{m}^{\dagger}-a_{0}\right)\right)+(b \rightarrow d, \zeta \rightarrow-\zeta)\right]+\hat{\rho}(\zeta) a_{0}
$$

with

$$
\hat{\rho}(\zeta)=\rho+2 \sum_{m=\frac{1}{2}}^{\Lambda}\left(\Delta_{m}(\zeta)+\Delta_{m}(-\zeta)-2 \Delta_{m}(0)\right)
$$

being a convergent function in $\Lambda$. In this way, matrix elements of the zero mode $a_{0}$ are rendered cutoff independent.

$\underline{5-P a r t i c l e ~ S e c t o r}$ I now examine what happens in a higher particle sector. The equation is represented schematically in Fig.(5) for the 5 particle sector when evaluating the 


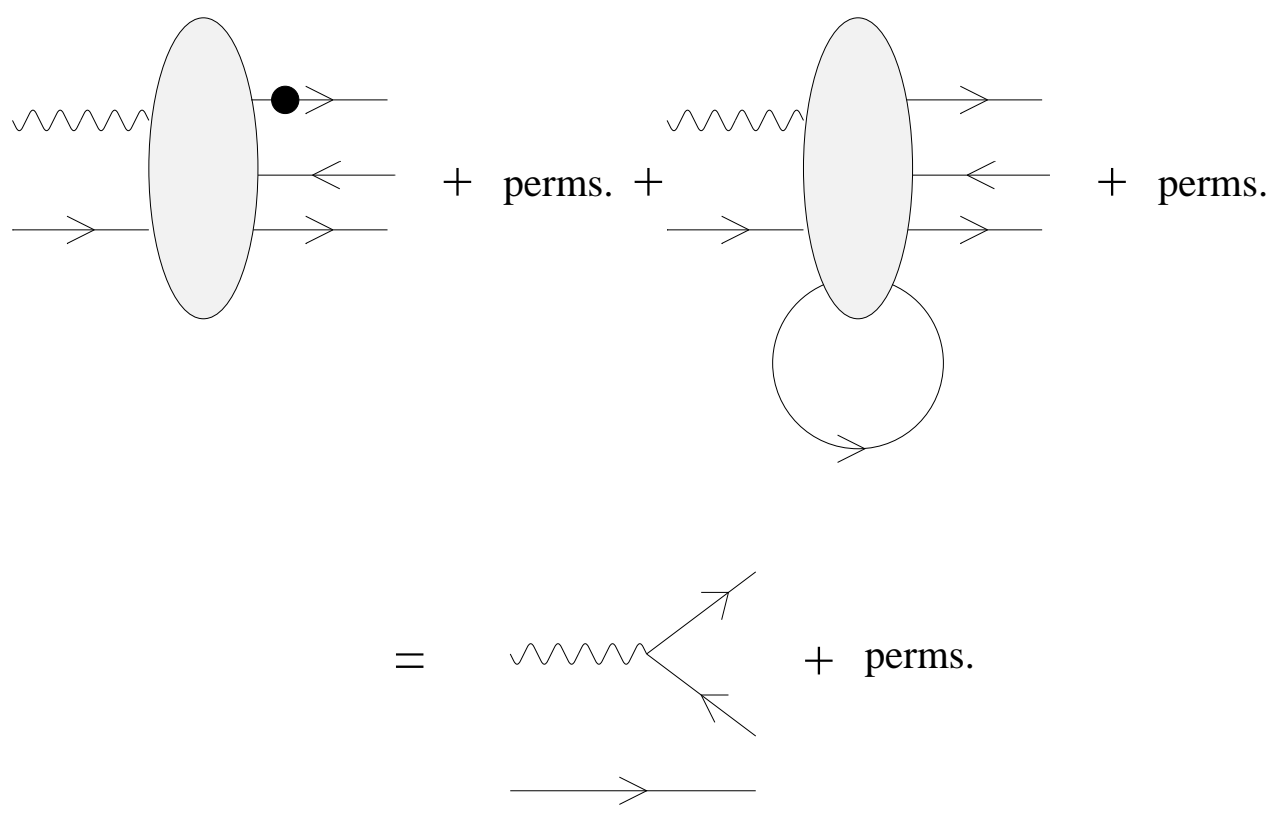

Figure 5: Some of the terms in one of the 5-particle sector of the constraint equation for states $\langle 0| a_{l} b_{m}$ and $b_{m_{1}}^{\dagger} d_{n}^{\dagger} b_{m_{2}}^{\dagger}|0\rangle$.

constraint between states $\langle 0| a_{l} b_{m}$ and $b_{m_{1}}^{\dagger} d_{n}^{\dagger} b_{m_{2}}^{\dagger}|0\rangle$. Evidently, the right hand side of the equation is non-zero only for particular configurations of momenta in the left hand side. Namely, for the case when the zero mode is not acting as essentially just a three-point operator with some spectators, the right hand side is zero. Thus, basically the constrained zero mode is a three-point vertex. However, because of terms such as the third and fourth in Fig.(3) it is not just the bare vertex. Rather the vertices are dressed.

Iteration to all orders. One can attempt solving the equation iteratively - essentially a weak coupling expansion. One would observe that to any order in the iteration the three separate contributions coming from respectively $\Gamma_{0}(\zeta)$ and $\Gamma_{1}( \pm \zeta)$ always decouple from each other. Mixing occurs solely via contractions between respectively $b$ with $b^{\dagger}$ and $d$ with $d^{\dagger}$. Diagrammatically, one can picture this in terms of precisely the terms in Fig.(3) that make the constraint operatorially non-trivial, namely the third and fourth terms which connect the different particle sectors. The way I proceed is to reexpress such terms as an effective three-body operator with an insertion, $\Sigma$, on the leg of the same colour as that in the loop. This is illustrated in Fig.(6). Evidently, no mixing can occur between $b$ and $d$ modes once the basic three-point vertices have been extracted. Thus the insertion can only arise as the sum to all orders of products of the basic propagator for a given value of 


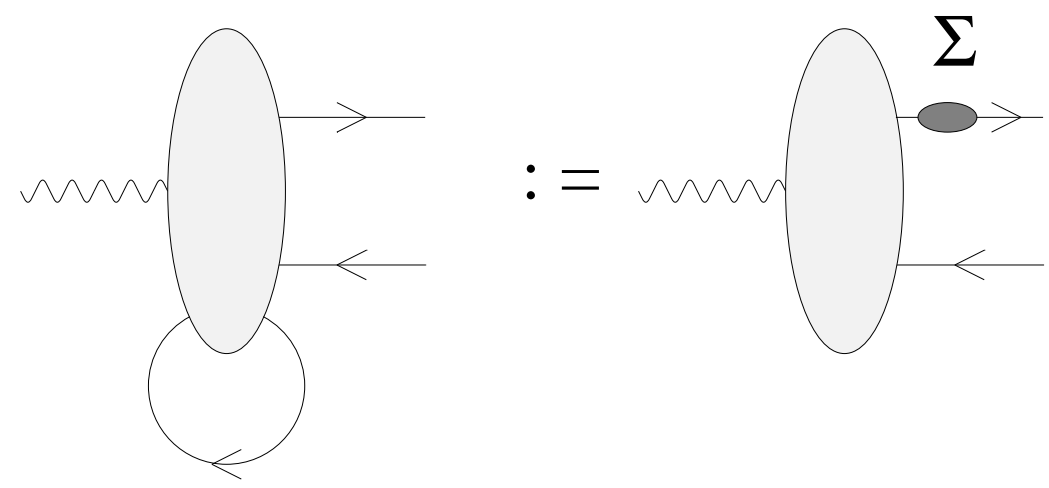

Figure 6: Identification of terms that connect different particle sectors of the constraint with an effective operator with insertion $\Sigma(\zeta)$. The analogous diagram can be drawn for the anticlockwise flow of momentum in the loop on the left hand side but where the insertion is made on the lower $d$-leg. Namely $\Sigma$ appears with argument $-\zeta$.

$\zeta$ as follows:

$$
\Sigma_{m}(\zeta)=\lim _{N \rightarrow \infty} \sum_{r=1}^{N}\left(\Delta_{m}(\zeta)\right)^{r}=\lim _{N \rightarrow \infty} \frac{1-\Delta_{m}(\zeta)^{N}}{1-\Delta_{m}(\zeta)} .
$$

Observe that for all but the lowest mode $m=1 / 2$, the propagator satisfies $\left|\Delta_{m}(\zeta)\right|<1$. Thus for the higher modes, Eq.(3.24) can be further simplified. However, for momentum $m=1 / 2$ the propagator will in general be 'large', and thus in turn the effect of $\Sigma_{1 / 2}(\zeta)$ will be even larger.

Solution. The above considerations motivate the following operator ansatz for the solution

$$
\begin{aligned}
a_{0} & =\sum_{l, m, n}\left[C_{0}^{l m n}(\zeta) \delta_{m+n}^{l}\left(a_{l}^{\dagger} b_{m} d_{n}+\text { h.c. }\right)\right. \\
& \left.+C_{1}^{l m n}(\zeta) \delta_{m+l}^{n}\left(b_{n}^{\dagger} b_{m} a_{l}+\text { h.c. }\right)+C_{1}^{l m n}(-\zeta) \delta_{m+l}^{n}(b \rightarrow d)\right] .
\end{aligned}
$$

This expression by construction satisfies the symmetries obeyed by the constrained zero mode. It is essentially a three-body operator, where the dressing of the vertices will be absorbed into the, as yet, arbitrary coefficients $C_{a}^{l m n}(\zeta)$. I insert Eq.(3.25) into Eq.(3.18) with the identification of Fig.(6) implemented. It suffices to consider the constraint in the three-particle space in order to solve for the coefficients, once one builds in the iteration to all orders in the insertion via Eq.(3.24). I represent the constraint as $L\left[a_{0}\right]=R$, where $L$ and $R$ denote the left- and right-hand sides of Eq.(3.18). I take the matrix elements

$$
\left\langle 0\left|a_{l} L\left[a_{0}\right] b_{m}^{\dagger} d_{n}^{\dagger}\right| 0\right\rangle=\left\langle 0\left|a_{l} R b_{m}^{\dagger} d_{n}^{\dagger}\right| 0\right\rangle
$$




$$
\left\langle 0\left|b_{n} L\left[a_{0}\right] b_{m}^{\dagger} a_{l}^{\dagger}\right| 0\right\rangle=\left\langle 0\left|b_{n} R b_{m}^{\dagger} a_{l}^{\dagger}\right| 0\right\rangle .
$$

The d-mode matrix element gives no additional information because of charge conjugation symmetry. The two equations are decoupled and one can straightforwardly determine the $C_{0}$ and $C_{1}$ coefficients in terms of the fundamental vertices and propagator

$$
\begin{aligned}
& C_{0}^{l m n}(\zeta)=\frac{\Gamma_{0}^{l m n}(\zeta)}{\mathcal{D}_{m, n}^{(0)}(\zeta)}, \mathcal{D}_{m, n}^{(0)}(\zeta) \equiv \Delta_{m}(\zeta)+\Delta_{n}(-\zeta)+\Sigma_{m}(\zeta)+\Sigma_{n}(-\zeta)+\hat{\rho}(\zeta), \\
& C_{1}^{l m n}(\zeta)=\frac{\Gamma_{1}^{l m n}(\zeta)}{\mathcal{D}_{m, n}^{(1)}(\zeta)}, \mathcal{D}_{m, n}^{(1)}(\zeta) \equiv \Delta_{m}(\zeta)+\Delta_{n}(\zeta)+\Sigma_{m}(\zeta)+\Sigma_{n}(\zeta)+\hat{\rho}(\zeta)
\end{aligned}
$$

With the coefficient functions determined one can now directly give the lowest particle matrix elements as

$$
\begin{aligned}
& \left\langle 0\left|a_{L} a_{0} b_{M}^{\dagger} d_{N}^{\dagger}\right| 0\right\rangle=\delta_{M+N}^{L} C_{0}^{L M N}(\zeta) \\
& \left\langle 0\left|b_{M_{1}} a_{0} b_{M_{2}}^{\dagger} a_{L}^{\dagger}\right| 0\right\rangle=\delta_{M_{2}+L}^{M_{1}} C_{1}^{L M_{2} M_{1}}(\zeta) \\
& \left\langle 0\left|d_{N_{1}} a_{0} d_{N_{2}}^{\dagger} a_{L}^{\dagger}\right| 0\right\rangle=\delta_{N_{2}+L}^{N_{1}} C_{1}^{L N_{2} N_{1}}(-\zeta) \text {. }
\end{aligned}
$$

Eqs.(3.25), (3.27) and (3.28) are the main results of this section.

\section{Revisiting the 'Adiabatic Potential'}

The Hamiltonian is the Poincaré generator $P^{-}$, given in Appendix $\mathrm{D}$ with other details from [17]. After removing a dimensionful factor $L(g / 4 \pi)^{2}$ one obtains the rescaled Hamiltonian, $H$. One seeks the ground state of the theory. If not for the gauge mode $\zeta$, the naive arguments leading to the trivial Fock vacuum being the true ground state would hold rigorously. Now in fact the ground state is some infinite superposition of $\zeta$ zero modes. One could try representing this in an oscillator basis but the true ground state is mostly likely some coherent state of these oscillator modes. As mentioned, I use instead a Schrödinger representation within the adiabatic approach of [17, 20]. The gauge mode $\zeta$ is frozen for the purposes of computing the ground state in the 'particle sector'. It is here the advantages of the light-cone become significant: the ground state is now the Fock vacuum. However, the tadpole terms that arise as one normal orders the Hamiltonian with respect to the trivial vacuum generate $\zeta$-dependent structures which become quantum operators once the gauge mode is unfrozen,

$$
H=: H[\zeta]:+V[\zeta]
$$

Equivalently, $V[\zeta] \equiv\langle 0|H[\zeta]| 0\rangle$. As shown in 17] and Appendix D, there is also a kinetic term for $\zeta$ surviving in $: H$ :. Thus, projecting in the Fock vacuum sector, we obtain a Hamiltonian

$$
H_{\zeta}=-4 \frac{d^{2}}{d \zeta^{2}}+V[\zeta]
$$


For later purposes it is convenient to break $V$ into three terms, coming respectively from the mass term, the constrained mode $a_{0}$ dependent term and the $a_{0}$ independent term

$$
V[\zeta]=V_{\rho}[\zeta]+V_{a}[\zeta]+V_{0}[\zeta]
$$

Solving the Schrödinger equation corresponding to $H_{\zeta}$ generates the ground state wave functional $\Psi_{0}(\zeta)$ allowing the introduction of the 'true' ground state as the tensor product state

$$
|\Omega\rangle \equiv \Psi_{0}[\zeta] \otimes|0\rangle
$$

Vacuum 'Degeneracy'. The only residue of the large gauge symmetries, Weyl reflections and central conjugations in the fundamental modular domain is the symmetry $\zeta \rightarrow-\zeta$, which is the basic symmetry of the potential. Thus there is an associated quantum number which labels the wavefunctions, namely symmetric $\Psi_{n}^{(+)}[\zeta]$ and antisymmetric $\Psi_{n}^{(-)}[\zeta]$ states. Thus identical copies of the particle spectrum can be built on either the vacuum described by $\Psi_{0}^{(+)}[\zeta]$ or $\Psi_{0}^{(-)}[\zeta]$ differing only by a fixed shift in the energy. The shift will be finite as the longitudinal interval length $L$ (factored out of $H_{\zeta}$ at this point) is kept finite. This is how the $Z_{2}$ analogue of $\theta$-vacua arise in this theory. The finiteness of the shift as $L \rightarrow \infty$ depends on the specific structure of $V$. In particular, the presence of an impenetrable barrier in the centre of the fundamental domain would bring the two lowest energies into exact degeneracy for any interval size $L$.

Renormalisation of $V_{\rho}+V_{0}$. The term $V_{0}$ was computed in [17]. Here I quote the relevant result:

$$
V_{0}[\zeta]=\sum_{n=\frac{1}{2}}^{\Lambda} \sum_{m=\frac{1}{2}}^{\Lambda}\left(\frac{u_{m}}{v_{n}}-\frac{v_{n}}{u_{m}}\right)^{2} w_{m+n}^{4}+\sum_{l=1}^{\Lambda+\frac{1}{2}} \sum_{m=\frac{1}{2}}^{\Lambda}\left[\left(\frac{w_{l}}{v_{m}}-\frac{v_{m}}{w_{l}}\right)^{2} v_{m+l}^{4}+\left(\frac{w_{l}}{u_{m}}-\frac{u_{m}}{w_{l}}\right)^{2} u_{m+l}^{4}\right]
$$

where $\Lambda$ is the (half-integer valued) cutoff in ultra-violet momenta. Using $u_{n}(\zeta)=$ $1 / \sqrt{n+\zeta}, v_{n}(\zeta)=1 / \sqrt{n-\zeta}$, and $w_{n}=1 / \sqrt{n}$ one can check this has the symmetry $\zeta \rightarrow-\zeta$. Substituting these coefficients, the expression is

$$
\begin{aligned}
V_{0}[\zeta] & =\sum_{n=\frac{1}{2}}^{\Lambda} \sum_{m=\frac{1}{2}}^{\Lambda} \frac{((m+\zeta)-(n-\zeta))^{2}}{(m+n)^{2}(m+\zeta)(n-\zeta)} \\
& +\sum_{l=1}^{\Lambda+\frac{1}{2}} \sum_{m=\frac{1}{2}}^{\Lambda}\left(\frac{(m-l+\zeta)^{2}}{l(m+\zeta)(l+m+\zeta)^{2}}+\frac{(m-l-\zeta)^{2}}{l(m-\zeta)(l+m-\zeta)^{2}}\right)
\end{aligned}
$$

Closer inspection reveals the sums to be logarithmic in the cutoff with a coefficient that depends on $\zeta$. It is this dependence I seek to extract. A useful trick is to add and subtract each of the three terms in Eq.(4.34) evaluated at $\zeta=0$. It is then a straightforward though 
tedious task to show that the expression can be reorganised as

$$
V_{0}[\zeta]=4 G[\zeta] \sum_{m=\frac{1}{2}}^{\Lambda} \frac{1}{m}+\text { Convergent }[\zeta]+\text { const }
$$

with

$$
G[\zeta]=\sum_{m=\frac{1}{2}}^{\Lambda}\left(\frac{1}{m+\zeta}+\frac{1}{m-\zeta}-\frac{2}{m}\right) .
$$

One recognises in $G$ the convergent functional $\hat{\rho}(\zeta)-\rho$, Eq.(3.23). One sees it comes in as the operator dependence of the logarithmic divergence.

The contributions of the mass term are more straightforward. After normal ordering, one extracts the structure

$$
V_{\rho}[\zeta]=\rho_{0}\left(\sum_{l=1}^{\Lambda} \frac{1}{l}+\sum_{m=\frac{1}{2}}^{\Lambda} \frac{1}{(m+\zeta)}+\sum_{m=\frac{1}{2}}^{\Lambda} \frac{1}{(m-\zeta)}\right)
$$

where $\rho_{0}$ was the bare (dimensionless) mass. In order to render the constraint equation and particle sector convergent and cutoff independent, the renormalised mass $\rho=\rho_{0}+$ $2 \sum_{m=1 / 2}^{\Lambda} \frac{1}{m}$ was introduced, Eq.(3.21). Substituting this into Eq.(4.37) gives

$$
V_{\rho}[\zeta]=-4 G[\zeta] \sum_{m=\frac{1}{2}}^{\Lambda} \frac{1}{m}+\rho G[\zeta]+\text { const. . }
$$

The $\zeta$-dependent but logarithmic divergent terms in Eq.(4.35) and Eq.(4.38) are equal but opposite in sign. Thus the same renormalisation holds respectively in the three separate cases of the two-particle bound state equation, the constraint equation, and in the $V_{0}+V_{\rho}$ parts of the potential.

As shown in [17], the shape of this part of the potential is flat within the fundamental domain, rising to positive infinity at the domain boundaries $\zeta= \pm \frac{1}{2}$. A careful study of the nature of the singular behaviour as $\zeta \rightarrow \pm \frac{1}{2}$ suggests it is not as strong as $1 /\left(\zeta \pm \frac{1}{2}\right)^{2}$, thus the potential at the domain boundaries appears to be penetrable. However, as argued in [25, 20, 21], the wavefunctions should be supplemented with a Jacobian determinant coming from the Faddeev-Popov structure in the present gauge. The wavefunctions are thus forced to vanish rigorously at the boundaries of the fundamental modular region. Thus the spectrum is approximately that of a square well which itself corresponds to that of pure glue $\mathrm{SU}(2)$ theory on a cylinder [28], with a sinusoidal ground state wavefunction. Either way, in the absence of the constrained mode there is no structure within the centre of the fundamental modular region. 


\section{Impact of the Constrained Mode}

I now include the effects of $a_{0}$. As shown in [17], there are terms in the Hamiltonian linear and quadratic in $a_{0}$, which were separated into $H_{\text {Constr }}=H_{\text {Constr }}^{(1)}+H_{\text {Constr }}^{(2)}$. They were respectively

$$
\begin{aligned}
& H_{\text {Constr }}^{(1)}=2 \sum_{k=\frac{1}{2}}^{\infty}\left[u_{k}^{3}\left(B_{k}^{\dagger} Q_{-}(k)+Q_{-}^{\dagger}(k) B_{k}\right)_{s}+v_{k}^{3}\left(D_{k}^{\dagger} Q_{+}(k)+Q_{+}^{\dagger}(k) D_{k}\right)_{s}\right] \\
& H_{\text {Constr }}^{(2)}=\sum_{k=\frac{1}{2}}^{\infty}\left[u_{k}^{2}\left(B_{k} B_{k}^{\dagger}+B_{k}^{\dagger} B_{k}\right)+v_{k}^{2}\left(D_{k} D_{k}^{\dagger}+D_{k}^{\dagger} D_{k}\right)\right]
\end{aligned}
$$

where the $Q_{ \pm}$arise from the $a_{0}$ independent parts of the currents and are given in Appendix D. The other operators are just $B_{k}=\left(a_{0} b_{k}+b_{k} a_{0}\right) / 2$ and $D_{k}=\left(a_{0} d_{k}+d_{k} a_{0}\right) / 2$ which arise because of symmetrisation of noncommuting operator products. I correspondingly consider the VEVs of the two terms separately. The linear term leads to

$$
\begin{aligned}
V_{1}[\zeta, \rho] & \equiv\left\langle 0\left|H_{\text {Constr }}^{(1)}\right| 0\right\rangle \\
& =\frac{1}{2} \sum_{l, m, n} \delta_{m+l}^{n}\left[R^{l m n}(\zeta)\left(\left\langle 0\left|a_{l} b_{m} a_{0} b_{n}^{\dagger}\right| 0\right\rangle+\left\langle 0\left|b_{n} a_{0} a_{l}^{\dagger} b_{m}^{\dagger}\right| 0\right\rangle\right)\right. \\
& \left.+R^{l m n}(-\zeta)(b \rightarrow d)\right]
\end{aligned}
$$

with

$$
R^{l m n}(\zeta)=u_{n}^{3}\left(\frac{w_{l}}{u_{m}}-\frac{u_{m}}{w_{l}}\right)
$$

which is actually part of the vertex function $\Gamma_{1}(\zeta)$. The quadratic term gives

$$
\begin{aligned}
V_{2}[\zeta, \rho] & \equiv\left\langle 0\left|H_{\text {Constr }}^{(2)}\right| 0\right\rangle \\
& =\sum_{m}\left[\Delta_{m}(\zeta)\left\langle 0\left|b_{m} a_{0}^{2} b_{m}^{\dagger}\right| 0\right\rangle+\Delta_{m}(-\zeta)\left\langle 0\left|d_{m} a_{0}^{2} d_{m}^{\dagger}\right| 0\right\rangle\right]
\end{aligned}
$$

One sees that the relevant contributions to the VEV come purely from the lowest particle sector matrix elements of $a_{0}$. For $V_{2}$ this follows after inserting a complete set of states between the two $a_{0}$ operators.

It is next a tedious task of inserting the solutions as given above. The most compact expression for the result is

$$
\begin{aligned}
\left\langle 0\left|H_{\text {Constr }}\right| 0\right\rangle=\quad & -2 \sum_{l=1}^{\Lambda+\frac{1}{2}} \sum_{m=\frac{1}{2}}^{\Lambda}\left[\frac{\left((m+\zeta)^{2}+l(m+l+\zeta)\right)}{l(m+\zeta)^{3}(m+l+\zeta)^{4} \mathcal{D}_{m, m+l}^{(1)}(\zeta)}\right. \\
& \left((m+\zeta)(m+l+\zeta)(m-l+\zeta)-2 \frac{\left((m+\zeta)^{2}+l(m+l+\zeta)\right)}{\mathcal{D}_{m, m+l}^{(1)}(\zeta)}\right] \\
& +[\zeta \rightarrow-\zeta]
\end{aligned}
$$


Evidently, for the high momentum terms in this expression the fluctuations with respect to $\zeta$ are small due to the restriction to the fundamental modular domain and so these contributions are essentially just constant with respect to $\zeta$. The dominant contributions to the $\zeta$ dependence are thus the lowest modes with $m=\frac{1}{2}$. Thus any structure that can appear is essentially coming from this one degree of freedom. In fact, I evaluated the double sum numerically using Mathematica. The behaviour of the potential was studied for different values of the physical dimensionless mass $\rho$. In addition, there is the parameter $N$ which defines the order to which the iterations in Eq.(3.24) is summed. Curiously, the convergence as $N \rightarrow \infty$ is very slow, but stability is achieved by the value $N=10^{6}$. As in the part of $V$ independent of $a_{0}$, the result here has a logarithmic dependence on the cutoff regulator. At this point I suppress the divergence by hand, but will return to the nature of this divergence in the following section.

Shape of the Constrained Part of the Potential. The final result for the renormalised potential, $V_{a}$, is represented in Fig.(7) for three values of the renormalised mass $\rho$. In the deep perturbative region $\rho \rightarrow \infty$ the potential is flat. However, as the mass $\rho$ is brought down, or coupling increased, the potential develops two degenerate minima. The barrier height appears to be at its maximum precisely at $\rho=0$. I now estimate its impact. A least square fit gives parabolae for the shape of the individual wells. For example, for the region $0<\zeta<0.2$ the curve can be well described by

$$
V_{a}^{(\mathrm{fit})}(\zeta)=54.9362 \zeta^{2}-11.2670 \zeta-0.0385
$$

Taking this together with the coefficient of 4 for the kinetic term in Eq.(4.30), one can estimate the lowest eigenstate if the given well were the complete potential: $E_{0} \sim 14.8 \gg$ 0.6. The latter number is the barrier height. Evidently even the lowest states are too energetic to feel the presence of the barrier.

\section{Open Renormalisation problem}

I now return to the remaining logarithmic divergences which in the previous calculation were suppressed by hand. The origin of these divergences is simple to see: in the vertex $\Gamma_{1}(\zeta)$ appears the factor $\left(\frac{w_{l}}{u_{n}}+\frac{u_{n}}{w_{l}}\right)$. This is contracted with the Kronecker delta function $\delta_{m+l}^{n}$ in the sums appearing in the potential. Evidently, for fixed momentum $m$ but $l$ large this factor scales as a constant

$$
\lim _{l \rightarrow \infty} \delta_{m+l}^{n}\left(\frac{w_{l}}{u_{n}}+\frac{u_{n}}{w_{l}}\right)=2 .
$$

Consequently, the large $l$ behaviour in the sums is not suppressed by a contribution from this term. 


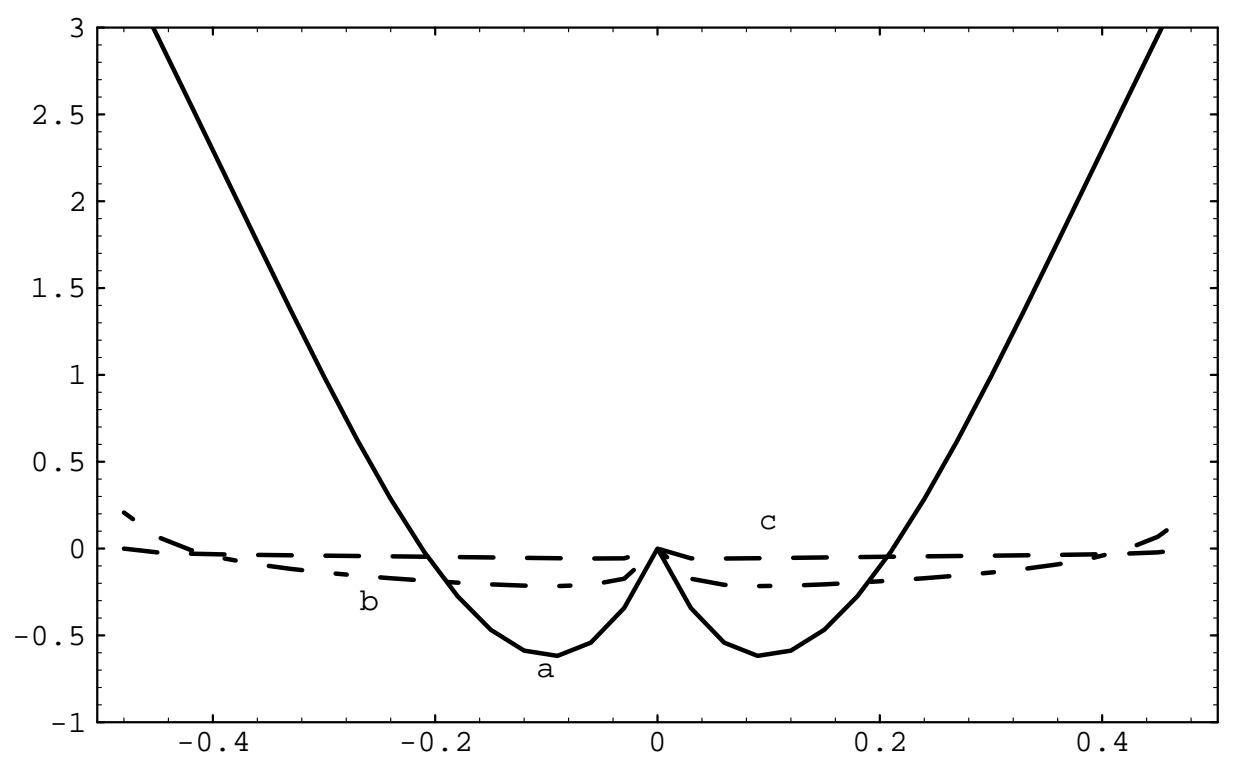

Figure 7: $V_{a}$ versus $\zeta$, namely the contribution of $a_{0}$ to the vacuum potential. The potential is a functional of $\zeta$ over the fundamental modular domain $-1 / 2<\zeta<1 / 2$. The curves are plotted for various values of renormalised mass $\rho$ : (a) $\rho=0$ (b) $\rho=20$ and (c) $\rho=100$. The potential has two minima whose depth increase with the coupling, namely with decreasing $\rho$. The wells are at their deepest for effectively massless 'gluons'. 


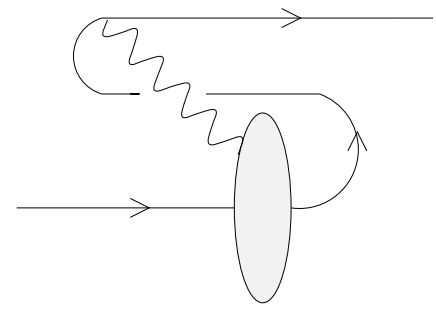

Figure 8: Example of a new 'Self-Induced Inertia' in the Hamiltonian, induced by substituting the constrained zero mode $a_{0}$ in the Hamiltonian and bringing the result to normal ordered form. Such a diagram contains logarithmically divergent coefficients.

One eventually realises this divergence is the tip of the iceberg when one begins reinserting the solution to the constraint equation into the Hamiltonian. New many-body operators will be induced, including two-body operators. An example is a term arising from a diagram of the form in Fig.(8) with the ' $R$ ' part of the $\Gamma_{1}$ vertex, Eq.(5.41). The expression corresponding to this diagram is

$$
H_{\text {new }} \propto \sum_{m=\frac{1}{2}}^{\Lambda} b_{m}^{\dagger} b_{m} \sum_{l=1}^{\Lambda+\frac{1}{2}} \sum_{n=\frac{1}{2}}^{\Lambda} R^{l m n}(\zeta) C_{1}^{l m n}(\zeta) \delta_{m+l}^{n} .
$$

One observes that the coefficient of the two-point operator matches the linear contribution of $a_{0}$ to the adiabatic potential in Eq.(5.40). The coefficient logarithmically diverges. This means that with the constrained mode eliminated, the two-particle bound state equation is once again ill-defined and must be renormalised over again by redefinition of the mass. There are many new terms contributing and the process of collecting them up is still underway. One is faced now with a subtlety: the constraint equation was rendered finite and cutoff dependent with the same renormalisation used in the twoparticle sector. Now that the latter would appear to have to change, it is not evident how the renormalisation of the constraint equation could be analogously modified. The main hurdle is conceptual rather than merely computational. It may be that the step of separating the two outstanding problems in light-cone quantisation - renormalisation and the zero modes - is too naive.

\section{Summary and Conclusions}

I restate the basic motivation for studying this theory: it was to understand the relationship between dynamical and constrained zero modes in a non-Abelian gauge theory with some non-trivial parton spectrum, and whether these modes contributed to the ex- 
pected vacuum structure of that theory. The model was that of two-dimensional SU(2) gauge theory coupled to massive scalar adjoint fields. In [17] was shown how a careful gauge-fixing leads to a linear constraint equation (as also occurs in $(3+1)$ for QCD [15]) for the zero mode of the real scalar field in this theory. This constraint, though at first glance complicated, had a systematic structure which could be interpreted in terms of propagators and vertices. A diagrammatic translation of the equation together with a consideration of the symmetries of the constrained mode lead to a method of solving the constraint. I then studied the role of the constrained zero mode in the effective potential governing the dynamical or 'gauge' zero mode. It turned out to generate a centrifugal barrier, the potential assuming a double-oscillator shape. Such a structure is to be expected from similar pictures in [29]. Evidently, including the constrained zero mode makes a qualitative difference in the potential of the dynamical zero mode in the direction of the expected result. However, the actual quantitative impact in this theory appears to be minimal. The outstanding problem is the completion of the renormalisation, which here was achieved simply by suppressing the logarithmic cutoff dependence by hand. A preliminary study of the further impact of the constrained mode in the Hamiltonian suggested that these divergences may go hand in hand with additional singular behaviour in the new self-induced inertias coming from the constraint. A detailed treatment of this problem is still in progress.

The conclusions are three-fold. Firstly, the zero mode has a structure which can be categorised diagrammatically. This is immensely useful for solving the relevant constraint

equations. Secondly, when one takes care to preserve all the modes of the present theory of massive adjoint scalars coupled to $\mathrm{SU}(2)$ glue, there is some additional structure in the theory originally treated by [16]. Within the framework of dimensional reduction of non-Abelian gauge theory, these structures are, at best, suggestive of vacuum and lowenergy phenomena of $\mathrm{QCD}(3+1)$. Thirdly, the problem of renormalisation in the light-cone approach may not be separable from that of the zero modes.

\section{Acknowledgement}

I am grateful to the following for intensive discussions and insightful suggestions: HansChristian Pauli, Steve Pinsky and Dave Robertson. I am especially grateful to Brett van de Sande for many discussions on nearly all aspects of this work, many insightful suggestions and patient guidance in the use of Mathematica. Rolf Bayer is thanked for assistance in the use of Xfig. This work was supported by a Max-Planck Gesellschaft Stipendium. 


\section{A Notation and Conventions}

Light-Cone Coordinates. I follow the convention of Kogut and Soper [30], with $x^{ \pm} \equiv$ $\left(x^{0} \pm x^{1}\right) / \sqrt{2}$.

Colour Helicity Basis. The colour helicity basis for $\mathrm{SU}(2)$ is defined in terms of the Pauli matrices $\sigma^{a}$ :

$$
\tau^{3}=\frac{1}{2} \sigma^{3}, \quad \tau^{ \pm} \equiv \frac{1}{2 \sqrt{2}}\left(\sigma^{1} \pm i \sigma^{2}\right)
$$

We can turn this into a vector space by introducing elements $x^{a}$ such that tilde quantities are defined with respect to the helicity basis, and untilded the usual Cartesian basis:

$$
x^{a}=\left(\begin{array}{c}
x^{1} \\
x^{2}
\end{array}\right) \quad \text { and } \quad \tilde{x}^{a}=\left(\begin{array}{c}
\tilde{x}^{1} \\
\tilde{x}^{2}
\end{array}\right)=\left(\begin{array}{c}
x^{+} \\
x^{-}
\end{array}\right) .
$$

The relation between the tilde and untilde basis can be written $\tilde{x}^{a}=\Lambda_{b}^{a} x^{b}$ and $x^{a}=\tilde{\Lambda}_{b}^{a} \tilde{x}^{b}$ where $\tilde{\Lambda}=\Lambda^{\dagger}$. With these elements we can construct the metric in terms of the tilde basis. Essentially we must demand the invariance of the inner product of any two vector space elements, $x^{a} y_{a}=\tilde{x}^{a} \tilde{y}_{a}$. Using the fact that the metric in the $a=1,2$ basis is just the Kronecker delta $\delta_{a b}$ and the transformed metric is $\tilde{\mathcal{G}}_{a b}=\tilde{\Lambda}_{a}^{c} \delta_{c d} \tilde{\Lambda}_{b}^{d}$. Thus

$$
\Lambda=\frac{1}{\sqrt{2}}\left(\begin{array}{rr}
1 & i \\
1 & -i
\end{array}\right) \quad \text { and } \quad \tilde{\mathcal{G}}_{a b}=\left(\begin{array}{cc}
0 & 1 \\
1 & 0
\end{array}\right) \text {. }
$$

The metric to raise and lower indices in the helicity basis becomes $x_{ \pm}=x^{\mp}$. The colour algebra looks formally like the Lorentzian structure in light-cone coordinates.

\section{B Gribov copies and the Wilson Loop}

Gribov Copies: Because of the torus geometry of the space and the non-Abelian structure of the gauge group, there remain large gauge transformations which are still symmetries of the theory [31, 32] despite the complete fixing of the theory with respect to small gauge transformations. These are generated by local SU(2) elements

$$
U\left(x^{-}\right)=\exp \left(-i n_{0} \pi \frac{x^{-}}{L} \tau_{3}\right), n_{0} \text { an even integer }
$$

which satisfy periodic boundary conditions. Another symmetry of the theory is $Z_{2}$ centre symmetry which here means allowing for antiperiodic $V$ or alternately $n_{0}$ odd. Both preserve the periodic boundary conditions on the gauge potentials. On the diagonal component of $A^{+} U$ generates shifts that are best expressed in terms of the dimensionless $z$, namely $z \rightarrow z^{\prime}=z+n_{0}$. On the scalar adjoint field and its momenta the effect of the 
transformation is

$$
\begin{aligned}
& \varphi_{3} \rightarrow \varphi_{3} \quad \text { and } \quad \varphi_{ \pm} \rightarrow \varphi_{ \pm} \exp \left(\mp i n_{0} \frac{\pi}{L} x^{-}\right) \\
& \pi^{3} \rightarrow \pi^{3} \text { and } \quad \pi^{ \pm} \rightarrow \pi^{ \pm} \exp \left( \pm i n_{0} \frac{\pi}{L} x^{-}\right)
\end{aligned}
$$

Colour Invariance of $z$ : The gauge mode $z$ can be written in terms of a colour singlet object. Construct the Wilson line for a contour $\mathrm{C}$ along the $x$ direction from $-L$ to $L$

$$
W=\operatorname{TrP} \exp \left(i g \int_{\mathrm{C}} d x_{\mu} \mathbf{A}^{\mu}\right)=\operatorname{TrP} \exp \left(i g \int_{-L}^{+L} d x \mathbf{A}^{+}\right)
$$

In the gauge used in this work, this is simply $W=\operatorname{Tr} \exp \left(2 i z \pi \tau^{3}\right)=2 \cos (2 \pi z)$. Thus, modulo the integers, $z=\frac{1}{2 \pi} \operatorname{arcos}\left(\frac{W}{2}\right)$. The integer shifts are the Gribov copies. Since $W$ is explicitly constructed in terms of a colour trace, $z$ is a colour singlet.

\section{Commutation Relations for Scalar Fields}

The commutators can be obtained, despite the presence of the constrained zero mode, from the Dirac procedure [34]. The result is well known, see for example [8], for scalar fields and can be directly taken over into the present theory. The quantum commutation relation at equal $x^{+}$for the normal modes is of the real scalar field is

$$
\left[\stackrel{n}{\varphi}_{3}(x), \pi^{3}(y)\right]_{x^{+}=y^{+}}=\frac{i}{2}\left[\delta\left(x^{-}-y^{-}\right)-\frac{1}{2 L}\right],
$$

where the $n$ above the field means the constrained zero mode $a_{0}$ has been removed and the last term ensures consistency for the commutator restricted to normal mode fields [35]. For the complex fields they are simply

$$
\left[\varphi_{-}(x), \pi^{-}(y)\right]_{x^{+}=y^{+}}=\left[\varphi_{+}(x), \pi^{+}(y)\right]_{x^{+}=y^{+}}=\frac{i}{2} \delta\left(x^{-}-y^{-}\right) .
$$

These relations demonstrate that there is no subtlety in the zero modes of the complex fields.

\section{Fourier transforms, Currents and the Hamiltonian}

The Discrete Fourier transforms of the scalar current components are defined by

$$
J_{3}^{+}\left(x^{-}\right) \equiv-\frac{1}{4 L} \sum_{k \in Z} e^{-i k \frac{\pi}{L} x^{-}} \widetilde{J}_{3}^{+}(k), \quad \text { and } \quad J_{ \pm}^{+}\left(x^{-}\right) \equiv-\frac{1}{4 L} \sum_{k \in H} e^{-i k \frac{\pi}{L} x^{-}} \widetilde{J}_{ \pm}^{+}(k)
$$

One can verify that $\left(\widetilde{J}_{3}^{+}(k)\right)^{\dagger}=\widetilde{J}_{3}^{+}(-k)$ and $\left(\widetilde{J}_{-}^{+}(k)\right)^{\dagger}=\widetilde{J}_{+}^{+}(-k)$. In the text, as in [17], the symmetrised operator products $B_{k} \equiv\left(a_{0} b_{k}+b_{k} a_{0}\right) / 2$ and $D_{k} \equiv\left(a_{0} d_{k}+d_{k} a_{0}\right) / 2$ were 
introduced. These carry the $a_{0}$ dependence in the currents $J_{ \pm}$leading to the construction of $Q$ operators

$$
\widetilde{J}_{3}^{+}(k) \equiv Q_{3}(k), \quad \widetilde{J}_{+}^{+}(k) \equiv Q_{+}(k)+\frac{D_{k}}{v_{k}}, \quad \text { and } \quad-\widetilde{J}_{-}^{+}(k) \equiv Q_{+}(k)+\frac{B_{k}}{u_{k}} .
$$

The $Q$-operators are thus $a_{0}$ independent. It suffices here to give the charge operator $Q_{-}$ explicitly

$$
\begin{aligned}
Q_{-}(k)=\sum_{n=1}^{\infty} \sum_{m=\frac{1}{2}}^{\infty} a_{n} b_{m}\left(\frac{w_{n}}{u_{m}}-\frac{u_{m}}{w_{n}}\right) \delta_{n+m}^{k} & +\sum_{n=1}^{\infty} \sum_{m=\frac{1}{2}}^{\infty} a_{n}^{\dagger} b_{m}\left(\frac{w_{n}}{u_{m}}+\frac{u_{m}}{w_{n}}\right) \delta_{n+k}^{m} \\
& -\sum_{n=1}^{\infty} \sum_{m=\frac{1}{2}}^{\infty} a_{n} d_{m}^{\dagger}\left(\frac{w_{n}}{v_{m}}+\frac{v_{m}}{w_{n}}\right) \delta_{m+k}^{n}(\mathrm{D}
\end{aligned}
$$

with $Q_{+}=\left(Q_{-}\right)^{\dagger}$.

The Hamiltonian is obtained from the energy-momentum tensor $\Theta^{\mu \nu}=2 \operatorname{Tr}\left(\mathbf{F}^{\mu \kappa} \mathbf{F}_{\kappa}{ }^{\nu}\right)-$ $g^{\mu \nu} \mathcal{L}$. At one level it is a simple expression, standard for $(1+1)$-dimensional gauge theories.

$$
P^{-}=\int_{-L}^{+L} d x^{-} \operatorname{Tr}\left(\partial_{+} \mathbf{A}^{+}-\mathbf{D}_{-} \mathbf{A}^{-}\right)^{2}=\int_{-L}^{+L} d x^{-} \operatorname{Tr}\left(\partial_{+} \mathbf{A}^{+} \partial_{+} \mathbf{A}^{+}-g^{2} \mathbf{J}^{+} \frac{1}{\mathbf{D}_{-}^{2}} \mathbf{J}^{+}\right)
$$

to which must be added the mass term $\mu_{0}^{2} \int d x^{-} \operatorname{Tr} \boldsymbol{\Phi}^{2}$. The above form merely expresses the dominance of the Coulomb potential in $(1+1)$ dimensions. However unpacking the currents $J^{+}$leads to nontrivial structure. The length dimension can be factored out by defining $H$ via $P^{-}=L(g / 4 \pi)^{2} H$. In terms of Fourier components, the gauge mode and Coulomb parts of $H$ can be rewritten as

$$
\begin{aligned}
H=-4 \frac{d^{2}}{d z^{2}}+\sum_{k=1}^{\infty} w_{k}^{4}\left(\widetilde{J}_{3}^{\dagger}(k) \widetilde{J}_{3}(k)+\widetilde{J}_{3}(k) \widetilde{J}_{3}^{\dagger}(k)\right) & +\sum_{k=\frac{1}{2}}^{\infty} v_{k}^{4}\left(\widetilde{J}_{+}^{\dagger}(k) \widetilde{J}_{+}(k)+\widetilde{J}_{+}(k) \widetilde{J}_{+}^{\dagger}(k)\right) \\
& +\sum_{k=\frac{1}{2}}^{\infty} u_{k}^{4}\left(\widetilde{J}_{-}^{\dagger}(k) \widetilde{J}_{-}(k)+\widetilde{J}_{-}(k) \widetilde{J}_{-}^{\dagger}(k)\right) .
\end{aligned}
$$

Relating $J$ operators to the $Q$ operators one can separate the constrained mode $a_{0}$ parts of $\mathrm{H}$ from the Fock sector. The reader is referred to [17] for more detail. When combined into the invariant mass-squared operator $M^{2}=2 P^{+} P^{-}$, with $P^{+}$the momentum operator, $L$ completely drops out in favour of $K=(L / \pi) P^{+}$, the harmonic resolution. The continuum limit $L \rightarrow \infty$ translates into $K \rightarrow \infty$ which is easily implementable in computer simulations. 


\section{References}

[1] S. Weinberg, Phys.Rev. 150 (1966) 1313.

[2] G.P. Lepage and S.J. Brodsky, Phys.Rev. D22 (1980) 2157.

[3] H.C. Pauli and S.J. Brodsky, Phys.Rev. D32 (1985) 1993; 2001.

[4] R.J. Perry, A. Harindranath and K. Wilson, Phys.Rev.Lett. 65 (1990) 2959.

[5] P.A.M. Dirac, Rev.Mod.Phys. 21 (1949) 392.

[6] M. Heyssler, A.C. Kalloniatis, Phys.Lett. B 354 (1995) 453.

[7] D.G. Robertson, Phys.Rev. D47 (1993) 2549.

[8] T. Maskawa and K. Yamawaki, Prog.Theor.Phys. 56 (1976) 270.

[9] T. Heinzl, S. Krusche, E. Werner and B. Zellermann, Phys.Lett. B272 (1991) 54,

[10] C.M. Bender, S.S. Pinsky, B. van de Sande, Phys.Rev. D48 (1993) 816.

[11] S.S. Pinsky and B. van de Sande, Phys.Rev. D49 (1994) 2001.

[12] S.S. Pinsky, B. van de Sande and J. Hiller, Phys.Rev. D51 (1995) 726.

[13] A. Borderies, P. Grangé, E. Werner, Phys.Lett. B 345 (1995) 458.

[14] M.A. Shifman, A.I. Vainshtein and V.I. Zakharov, Nucl. Phys. B147 (1979) 385.

[15] V.A. Franke, Yu.A. Novozhilov, and E.V. Prokhvatilov, Lett.Math.Phys. 5 (1981) 239;437; Light-Cone Quantization of gauge Theories with periodic Boundary Conditions in Dynamical Systems and Microphysics, Academic Press, 1982, p.389-400.

[16] K. Demeterfi, I.R. Klebanov, and G. Bhanot, Nucl.Phys. B418 (1994), 15; G. Bhanot, K. Demeterfi, and I.R. Klebanov, Phys.Rev. D48 (1993) 4980.

[17] H.C. Pauli, A.C. Kalloniatis, S.S.Pinsky, Towards Solving QCD - The Transverse Zero Modes in Light-Cone Quantisation. hep-th/9509020. To appear in Phys. Rev. D; S.S. Pinsky, A.C. Kalloniatis, Light-Front QCD(1+1) Coupled to Adjoint Scalar Matter. Submitted to Phys. Lett. B.

[18] H.-C. Pauli, R. Bayer, Towards Solving QCD in Light-Cone Quantization - On the Spectrum of the Transverse Zero Modes for SU(2) Heidelberg Preprint MPI H-V321995.

[19] N.S. Manton, Ann.Phys.(N.Y.) 159 (1985) 220. 
[20] F. Lenz, M. Shifman, M. Thies, 1994.

[21] F. Lenz, H.W.L. Naus, M. Thies, Ann. Phys. (N.Y.) 233 (1994) 317.

[22] A.C. Kalloniatis and H.C. Pauli, Z.Phys. C63 (1994) 161.

[23] A.C. Kalloniatis and D.G. Robertson, Phys.Rev. D50 (1994) 5262.

[24] A.C. Kalloniatis, H.C. Pauli, and S.S. Pinsky, Phys.Rev. D50 (1994) 6633.

[25] M. Lüscher, Nucl.Phys. B219 (1983) 233.

[26] P. van Baal, Nucl.Phys. B369 (1992) 259.

[27] A. C. Kalloniatis, Solving the Zero Mode Problem of QCD, in Proceedings of Workshop on Hadrons and Light-Front QCD, Zakopane, Ed. St. Glazek, 1994.

[28] J.E. Hetrick, Nucl.Phys. B30 (1993) 228;Int.J.Mod. Phys. A9 (1994) 3153.

[29] P. van Baal, J. Koller, Ann.Phys.(N.Y.) 174 (1987) 299.

[30] J.B. Kogut, D.E. Soper, Phys.Rev. D1 (1970) 2901.

[31] V.N. Gribov, Nucl.Phys. B 139 (1978) 1.

[32] I.M. Singer, Commun.Math.Phys. 60 (1978) 7.

[33] S. Dalley, private communication.

[34] P.A.M. Dirac, Lectures on Quantum Mechanics. (Academic Press, New York, 1964)

[35] A. C. Kalloniatis, H.-C. Pauli, Z.Phys. C60 (1993) 255. 